\title{
Predation of migratory Little Stint (Calidris minuta) by Barbary Falcon (Falco pelegrinoides) is dependent on body mass and duration of stopover time
}

\author{
Reuven Yosef • Bartłomiej Gołdyn • \\ Piotr Zduniak
}

Received: 28 September 2010/Accepted: 12 November 2010/Published online: 11 December 2010

(C) The Author(s) 2010. This article is published with open access at Springerlink.com

\begin{abstract}
Shorebird (Charadriiformes) migration phenology is critically synchronised with prey availability at traditional staging sites that allows the birds to stage and complete the migrations. These stopovers, usually in large concentrations, make the migrants vulnerable to local predators. The body mass gained or maintained is considered to be the result of the trade-off between the risks of starvation and predation. Theoretically, heavier birds should be less adept at escaping predators, and experimental evidence showed that flight performance is impaired in heavier birds. During three migration seasons we searched pellets and prey remains taken by a pair of Barbary Falcons (Falco pelegrinoides). We discovered that many pellets contained rings from the ringing program in Eilat, Israel, and the overwhelming majority belonged to Little Stints (Calidris minuta). We checked if the body mass and length of the stopover as expressed by ringing status of the Little Stints in a particular migration season was related to the risk of predation by Barbary Falcons. We found the chance that a
\end{abstract}

R. Yosef

International Birding and Research Centre in Eilat,

P. O. Box 774, 88000 Eilat, Israel

B. Gołdyn

Department of General Zoology, Faculty of Biology,

Adam Mickiewicz University, Umultowska 89,

61-614 Poznań, Poland

P. Zduniak $(\bowtie)$

Department of Avian Biology and Ecology,

Faculty of Biology, Adam Mickiewicz University,

Umultowska 89, 61-614 Poznań, Poland

e-mail: kudlaty@amu.edu.pl heavier bird would be predated was lower than that of a lighter individual, and that Stints retrapped during the same migration season were significantly more endangered by predation than those recorded only once.

Keywords Body mass $\cdot$ Calidris minuta $\cdot$ Falco pelegrinoides $\cdot$ Migration $\cdot$ Predation

\section{Introduction}

Shorebirds (Charadriiformes) are mostly temperate and Arctic breeding species that migrate to winter in tropical or subtropical regions. Their life history is constrained by the fact that they have a very restricted window of time within which they must arrive at their breeding grounds, reproduce and fledge young, and leave the breeding area prior to the onset of inclement weather and subsequent lack of resources. Hence, their migration phenology is critically synchronised with prey availability at traditional staging sites that will allow them to stage and complete the migrations (Wilson 1991).

Shorebirds typically migrate in a series of long-distance flights with traditional staging areas between flights (Myers et al. 1987). To complete migration, shorebirds have to deposit large quantities of fat by feeding rapidly at these staging areas. Their high metabolic rates, often higher than those predicted on scaling curves of metabolic rates of body mass (Kersten and Piersma 1987), require that waders accumulate fat deposits that may at times be greater than $50 \%$ of their lean body mass (Castro and Myers 1990). To achieve this, they have to remain for an extended period of time at the staging areas. These stopovers, usually in large concentrations, make the migrants vulnerable to local predators (e.g., Dekker 2003). 
The body mass gained or maintained is considered to be the result of the trade-off between the risks of starvation and being unable to continue or complete the migration, and predation (e.g., Gosler et al. 1995; Lilliendahl 1997; Fransson and Weber 1997; Swaddle and Lockwood 1998; Burns 2003). Theoretically, heavier birds should be less adept at escaping predators (Hedenström 1992); and experimental evidence has shown that flight performance is impaired in heavier birds (e.g., Witter et al. 1994; Kullberg et al. 1996; Lind et al. 1999). Witter and Cuthill (1993) suggested that increased predation may not only be due to increased body mass, but also due to increased foraging rates resulting in reduced vigilance, thus increasing vulnerability to predation. Evidence from field studies shows that prey species have lower body mass during periods of high predation (Gosler et al. 1995) or in response to a perceived predation risk (Lilliendahl 1997; Fransson and Weber 1997). On the other hand, experimental study by Jones et al. (2009) suggests that heavier birds allocate more attention to antipredator behaviour by modifying interscan intervals, resulting in faster response times to a predator. Kenward (1978) found that Goshawk (Accipiter gentilis) prey more frequently on Wood Pigeons (Columba palumbus) in poor body condition and usually on those which foraged singly. Predation of weaker individuals was especially marked if a flock took off when the Goshawk was further away and it had to chase the flock so vulnerable birds could be singled out.

During three migration seasons, we regularly searched for pellets and remains of prey taken by the Barbary Falcons at the base of the electricity pylon. We discovered that many pellets contained rings from our ringing program and the overwhelming majority belonged to Little Stints (Calidris minuta).

In order to verify the "mass-dependent predation risk" (Jones et al. 2009), the aim of our study was to check if risk of predation to Little Stints by Barbary Falcons was related to body mass and duration of stay at the stopover site as expressed by the ringing status in any particular migration season.

We hypothesized that heavier Little Stints would be more easily caught than lighter individuals (e.g., Witter et al. 1994; Kullberg et al. 1996; Lind et al. 1999). We reasoned that the open terrain of the salt ponds, proximity of the hunting and feeding perch from which the falcons initiated their attacks, and the slower flight capability of heavier stints would make them more vulnerable to predation than lighter conspecifics. We also hypothesized that individuals required to stay longer at the staging area in Eilat, and therefore subject to retrapping, would be more exposed to the risk of predation than those that made shortterm migratory stops.

\section{Materials and methods}

\section{Study species}

The Little Stint is a small shorebird that breeds in the tundra habitats of the high Arctic; it is one of the most numerous wader species migrating across much of the west Palearctic (Snow and Perrins 1998). In Israel, the species is an abundant passage migrant and fairly common winter visitor over most low-lying areas (Shirihai 1996). The Barbary Falcon is a resident in the Eilat region and is observed year round. They usually hunt solitarily, although pairs or family groups are occasionally seen foraging together (pers. obs.).

During the normal work at the International Birding and Research Center in Eilat (IBRCE) ringing station at the Bird Sanctuary, we observed a pair of Barbary Falcons (Falco pelegrinoides) that frequented the salt ponds for prey. The majority of stints taken were staging shorebirds trapped in walk-in traps as part of the banding program. The falcons' favourite lookout site and feeding perch was a $12 \mathrm{~m}$ high electricity pylon. This was situated in a barbed wire enclosure preventing access by humans and terrestrial predators, located such that it offers an unobstructed view of the waterline of the salt pond. The location of the pylon allowed the falcons to stoop directly at the prey, without having to circle above them-which often disclosed their intentions-and to surprise the waders while they were foraging.

\section{Study site}

Eilat, Israel, is a land bridge connecting three continentsAfrica, Asia, and Europe-and is located along the eastern Palearctic flyway used by birds wintering in tropical Africa and breeding in Eurasia. For migrating birds, Eilat $\left(29^{\circ} 33^{\prime} \mathrm{N}, 34^{\circ} 57^{\prime} \mathrm{E}\right)$ is located in front of (autumn) or behind (spring) the Sahel, Sahara, and Sinai Desert crossings, at the edge of almost 2,000 km of continuous desert (Yosef and Tryjanowski 2002). To the northeast lies the Syrian Desert, and to the east the Arabian Desert (Yosef 1997). Eilat, including the IBRCE bird reserve, is an oasis along the desert route and is a critical stopover and refuelling site for many migrant species (e.g., Tryjanowski and Yosef 2002; Yosef and Chernetsov 2004, 2005; Zduniak and Yosef 2008; Yosef and Weinman 2010).

Catching and ringing birds, and collecting pellets and bird remains

During two spring (February-May in 2001, 2002) and one autumn (September-December in 2002) migration seasons, 929 Little Stints were ringed (196, 296, 437, respectively) 
and 56 retrapped $(15,7,20$, respectively), i.e., individuals ringed in previous seasons were recaptured. Birds were caught in walk-in traps located on the salinas of the IBRCE. Each bird caught was aged and classified into four age classes: juveniles and adults in autumn and immature (in the second calendar year) and adults in the spring (EURING codes 3, 4 and 5, 6, respectively). Body mass of each individual was determined with an electronic digital scale to the nearest $0.1 \mathrm{~g}$.

We conducted a weekly search at the base of the pylon and collected all the bird remains, rings and pellets discarded by the falcons during the preceding week. In total we found 73 rings (19 in spring 2001, 26 in spring 2002, 28 in autumn 2002) which were later identified in the data set of the IBRCE as those placed on Little Stints.

Data processing and analysis

From the whole data set from the three migration seasons, we analysed records from the days when Little Stints were recorded alive for the last time and were later predated by falcons. Overall there were 539 records from 48 days (101 in spring 2001, 188 in spring 2002, 250 in autumn 2002), including 73 records of birds captured by the falcons and 466 records of Little Stints that were not known to be predated. Using such selected data we analysed the effect of body mass, age (juvenile/immature and adult) and ringing status (only one record or retrap in that migration season) of Little Stints for probability of being captured by the Barbary Falcons using canonical variate analysis (CVA, ter Braak and Šmilauer 2002). In this model the records were divided in two groups (predated and not predated Little Stints) using two binary variables regarded as dependent in the analysis. Body mass (in grams, linear variable), age (categorical) and retrap (binary variable) for each individual were included in the model as explanatory variables. Furthermore, to reduce the possible influence of sampling time, we included into the model as covariates the year, migration season and the day of sampling in each season. Moreover, records from each ringing day were grouped to form blocks of data organised as a time series. The statistical significance of the explanatory variables included in the model was tested using the Monte Carlo permutation test set for 5,000 permutations. The permutations were performed in cyclic shifts restricted to the above-mentioned blocks of data. This way the analysis was performed with respect to the groups of potential prey items during each successful hunting event.

On the resulting CVA ordination diagram the first ordination axis depicts the relation between dependent and explanatory variables (Fig. 1). Positions of arrowheads (body mass and age) and circle (retrap) are related to the likelihood of being predated or not predated (triangles).
The second dimension (axis II) reflects how the model fits the data. The more the variance in dependent variables is explained, the closer are the triangles to the first axis. After we performed the CVA analysis, we used the locally weighted scatterplot smoothing (LOESS) method to check how the most significant explanatory variable (body mass) is related to the chance of being predated or not. Isolines $($ alpha $=10)$ from this analysis representing the body mass classes (18-25 g in $1 \mathrm{~g}$ intervals) were fitted to the CVA ordination space on the diagram from the previous analysis. The CVA and isoline fitting were performed using CANOCO for Windows 4.5 software package (ter Braak and Šmilauer 2002; Lepš and Šmilauer 2003).

\section{Results}

CVA shows that the chance that a heavier bird would be predated was lower than that of a lighter individual $(F=11.52, p=0.004$, Fig. 1$)$. The LOESS places the centroids of predated and not predated individuals in the ranges of 19-20 and 22-23 g, respectively (Fig. 1, $R^{2}=0.19$ )

The second factor determining the chance to be caught by a falcon is the period of time spent at the stopover site. Stints retrapped during the same migration season were significantly more endangered by predation by the falcon than those recorded only once $(F=31.15, p<0.001$, Fig. 1). Furthermore, age of the potential prey did not significantly influence the chance of being predated $(F=4.05, p=0.054)$. The final CVA model was significant at $p=0.0002(F=47.59$; eigenvalue $=0.081$; species-environment correlation $=0.286$ ).

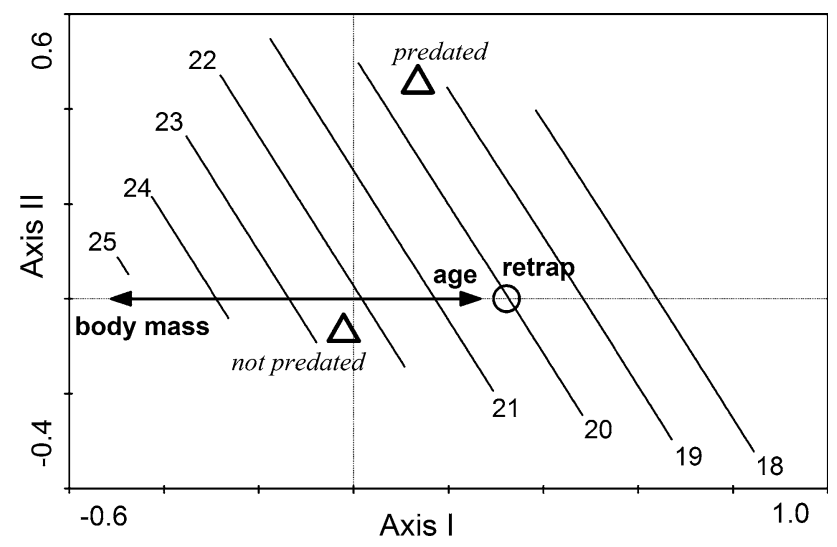

Fig. 1 Canonical variate analysis ordination diagram for the effect of body mass (arrow and isolines), age (arrow) and ringing status (retraps marked with circle) of the Little Stints on probability of being predated or not (triangles) by Barbary Falcons. Isolines representing values of body mass were fitted to the ordination space of the diagram after the CVA analysis and were determined using the LOESS method $\left(R^{2}=0.19\right)$ 


\section{Discussion}

Our results do not corroborate our first hypothesis that the chance of a heavier bird being preyed upon by a falcon is greater than that of lighter individuals. The results do not substantiate the findings of Hedenström (1992), Witter et al. (1994), and Kullberg et al. (1996) who considered heavier birds to be more vulnerable to predators owing to obesity and impaired flight ability. Owing to the lack of observations in parallel to the collection of the rings during the study, we are unable to verify the findings of impaired flight or the advantage offered the falcons by the short distance between their perch and the foraging waders. It remains unclear if lighter birds are more vulnerable to the predators as a function of their reduced vigilance owing to their need for increased food intake (cf. Gosler et al. 1995; Lilliendahl 1997; Fransson and Weber 1997) and slower response times to predators (Jones et al. 2009). Even though Barbary Falcons have not been reported to scavenge or feed on carrion, it is possible that some of the lightest birds died prior to being collected by the falcons. However, we consider this possibility as negligible, because on all occasions that we or other birdwatchers observed the falcons, they caught their prey by stooping or in a direct flight from the perch. Little Stints establish feeding territories along the water line during the day throughout the staging period in Eilat, and do not flock, which prevented us from understanding the effect of flock size and body condition on their susceptibility to predation.

Studies on another wader, the Redshank (Tringa totanus), showed that individuals which do not engage in escape flight behaviour (Cresswell 1993) or those closer to cover (Whitfield 2003) are more likely to be captured by a predator, suggesting the importance of early detection and an appropriate escape response (Jones et al. 2009).

Further, an important factor influencing the speed of predator detection is habitat structure (Whittingham et al. 2004; Devereux et al. 2006). In our case, the advantage is clearly with the falcons-open salinas, no escape routes or shelter, and foraging singly are all disadvantageous to the waders.

Thus, in the case of waders foraging at the waterline of the obstacle-free salt ponds, slow detection of aerial predators seems to be the most important feature disallowing sufficient time to respond and escape. Decrease of body mass in the Little Stint increases the probability of being caught by a Barbary Falcon because lighter birds forage more and faster with reduced vigilance, resulting in slower predator detection (Jones et al. 2009).

Kenward (1978) suggested that individuals below the minimum threshold body mass should try to feed as fast as possible, even at the cost of being risk-prone, in order to attain the viable body mass that would allow them to join a flock, resulting in improved risk-averse group vigilance. This strategy would be advantageous to Little Stints that are time stressed in order to reach the wintering areas in sub-Saharan Africa in the fall and/or the tundra breeding grounds in the spring.

Apart from body mass, the second factor that determined the chance of being preyed upon by a falcon was the period of time spent at the stopover site during the migration season (e.g., Markovets and Yosef 2005). Likewise, the longer a bird stayed at the stopover site, the greater the chance of it being caught by the falcons. Hence, Little Stints at our study site should feed and gain body mass as fast as possible; speed of foraging is a trade-off between body mass gain and the level of predation risk. The best strategy appears to be fast and safe feeding, but this can be achieved only by high quality individuals, which can forage faster and therefore have a higher scan rate of surroundings. These birds are more able to detect predators when compared to less fit individuals (Cresswell et al. 2003; Jones et al. 2009).

This study supports the idea that the body mass and duration of stay at the stopover site during migration by waders determines vulnerability to avian predators. Little Stints with lower body mass and those that stayed longer at the stopover site were more exposed to the Barbary Falcons. Moreover, this study is an example of the advantage of searching for and collecting pellets and bird remains in and around ringing stations, providing an indirect understanding of the birds' ecology, behaviour and survivability.

Acknowledgments We thank Mrs. L. Mitchell and J. Scott for their help in the field. Susan Craig and two anonymous reviewers improved earlier drafts of the manuscript.

Open Access This article is distributed under the terms of the Creative Commons Attribution Noncommercial License which permits any noncommercial use, distribution, and reproduction in any medium, provided the original author(s) and source are credited.

\section{References}

Burns JC (2003) Relationship of Calidris sandpiper wing shape with relative fuel load and total migration distance. Auk 120:827-835

Castro G, Myers JP (1990) Validity of predictive equations for total body fat in sanderlings from different nonbreeding areas. Condor 92:205-209

Cresswell W (1993) Escape responses by redshanks, Tringa totanus, on attack by avian predators. Anim Behav 46:609-611

Cresswell W, Quinn JL, Whittingham MJ, Butler S (2003) Good foragers can also be good at detecting predators. Proc R Soc Lond B 270:1069-1076

Dekker D (2003) Peregrine falcon predation on dunlins and ducks and kleptoparasitic interference from bald eagles wintering at Boundary Bay, British Columbia. J Raptor Res 37:91-97

Devereux CL, Whittingham MJ, Fernandez-Juricic E, Vickery JA, Krebs JR (2006) Predator detection and avoidance by starlings 
under differing scenarios of predation risk. Behav Ecol 17: 303-309

Fransson T, Weber TP (1997) Migratory fuelling in blackcaps (Sylvia atricapilla) under perceived risk of predation. Behav Ecol Sociobiol 41:75-80

Gosler AG, Greenwood JJD, Perrins C (1995) Predation risk and the cost of being fat. Nature 377:621-623

Hedenström A (1992) Flight performance in relation to fuel loads in birds. J Theor Biol 158:535-537

Jones KA, Krebs JR, Whittingham MJ (2009) Heavier birds react faster to predators: individual differences in the detection of stalking and ambush predators. Behav Ecol Sociobiol 63:1319-1329

Kenward RE (1978) Hawks and doves: factors affecting success and selection in goshawk attacks on woodpigeons. J Anim Ecol 47:449-460

Kersten M, Piersma T (1987) High levels of energy expenditure in shorebirds: metabolic adaptations to an energetically expensive way of life. Ardea 75:175-187

Kullberg C, Fransson T, Jakobsson S (1996) Impaired predator evasion in fat blackcaps (Sylvia atricapilla). Proc R Soc Lond B Biol Sci 263:1671-1675

Lepš J, Šmilauer P (2003) Multivariate analysis of ecological data using CANOCO. Cambridge University Press, Cambridge

Lilliendahl K (1997) The effect of predator presence on body mass in captive greenfinches Carduelis chloris. Anim Behav 53:75-81

Lind J, Fransson T, Jakobsson S, Kullberg C (1999) Reduced take-off ability in robins (Erithacus rubecula) due to migratory fuel load. Behav Ecol Sociobiol 46:65-70

Markovets M, Yosef R (2005) Phenology, duration and site fidelity of wintering bluethroat (Luscinia svecica) at Eilat, Israel. J Arid Environ 61:93-100

Myers JP, Morrison RIG, Antaz PA, Harrington BA, Lovejoy TE, Sallaberry M, Senner SE, Tarak A (1987) Conservation strategy for migratory species. Am Sci 75:19-26

Shirihai H (1996) The birds of Israel. Academic Press, London

Snow DW, Perrins CM (1998) The birds of the Western Palearctic. Concise ed. Vol 1. Non-passerines. Oxford Univ Press, Oxford

Swaddle JP, Lockwood R (1998) Morphological adaptations to predation risk in passerines. J Avian Biol 29:172-176

Ter Braak CJF, Šmilauer P (2002) CANOCO reference manual and CanoDraw for Windows user's guide: software for canonical community ordination (version 4.5)-microcomputer power. Microocomputer Power, Ithaca

Tryjanowski P, Yosef R (2002) Differences between the spring and autumn migration of the red-backed shrike Lanius collurio: record from the Eilat stopover (Israel). Acta Ornithol 37:85-90

Whitfield DP (2003) Redshank Tringa totanus flocking behaviour, distance from cover and vulnerability to sparrowhawk Accipiter nisus predation. J Avian Biol 34:163-169

Whittingham MJ, Butler S, Quinn JL, Cresswell W (2004) The effect of limited visibility on vigilance behaviour and speed of predator detection: implications for the conservation of granivorous passerines. Oikos 106:377-385

Wilson WH Jr (1991) The foraging ecology of migratory shorebirds in marine soft-sediment communities: the effects of episodic predation on prey populations. Am Zool 31:840-848

Witter MS, Cuthill IC (1993) The ecological cost of avian fat storage. Phil Trans R Soc Lond B 340:73-92

Witter MS, Cuthill IC, Bonser RH (1994) Experimental investigations of mass-dependent predation risk in the European starling, Sturnus vulgaris. Anim Behav 48:201-222

Yosef R (1997) Clues to the migratory routes of the eastern flyway of the Western Palearctics-ringing recoveries at Eilat, Israel (ICiconiiformes, Charadriiformes, Coraciiformes, and Passeriformes). Die Vogelwarte 39:131-140

Yosef R, Chernetsov N (2004) Stopover ecology of migratory Sedge Warblers (Acrocephalus schoenobaenus) at Eilat, Israel. Ostrich 75:52-56

Yosef R, Chernetsov N (2005) Longer is fatter: body mass changes of migrant Reed Warblers (Acrocephalus scirpaceus) staging at Eilat, Israel. Ostrich 76:142-147

Yosef R, Tryjanowski P (2002) Migratory masked shrikes Lanius nubicus staging at the desert edge: phenology, and sex- and agerelated differences in body mass. Ostrich 73:162-165

Yosef R, Weinman A (2010) Differential stopover ecology of blackcaps (Sylvia atricapilla) by sex and age at Eilat, Israel. J Arid Environ 74:360-367

Zduniak P, Yosef R (2008) Age and sex determine the phenology and biometrics of migratory common quail (Coturnix coturnix) at Eilat, Israel. Ornis Fennica 85:37-45 